\title{
High-order nonlinearities in the motion of a trapped atom
}

\author{
S. Wallentowitz and W. Vogel \\ Arbeitsgruppe Quantenoptik, Fachbereich Physik, Universität Rostock, Universitätsplatz 3, D-18051 Rostock, Germany \\ P. L. Knight \\ Blackett Laboratory, Imperial College, London SW7 2BZ, United Kingdom
}

(Received 10 June 1998)

\begin{abstract}
We study the counterpart to the multiphoton down-conversion in the quantized motion of a trapped atom. The Lamb-Dicke approximation leads to a divergence of the mean motional excitation in a finite interaction time for $k$-quantum down-conversions with $k \geqslant 3$, analogous to the situation in the parametric approximation of nonlinear optics. We show that, in contrast to the Lamb-Dicke approximation, the correct treatment of the overlap of the atomic center-of-mass wave function and the driving laser waves leads to a proper dynamics without any divergence problem. That is, the wavy nature of both matter and light is an important physical property that cannot be neglected for describing the motional dynamics of a trapped atom, even for small Lamb-Dicke parameters. [S1050-2947(99)00301-7]
\end{abstract}

PACS number(s): 42.50.Vk, 32.80.Lg, 42.65.-k, 03.65.- -w

\section{INTRODUCTION}

When the susceptibility of a medium interacting with an electromagnetic field of optical frequency depends strongly on the electric-field amplitude, one enters the domain of nonlinear optics. Nonlinear couplings of electric fields of different frequencies usually emerge from an expansion of the susceptibility in terms of the electric-field amplitude. Prominent examples of such nonlinear couplings are secondharmonic generation or two-photon down-conversion, which are due to a second-order susceptibility $\chi_{2}$. Nonlinear crystals have been successfully used to produce squeezed quantum states of light via a two-photon down-conversion. The extension of two-photon down conversion to an arbitrary $k$ photon process, where $k>2$ has also been studied. While this might be viewed as a natural generalization of the secondharmonic generation or the two-photon down-conversion, it has been shown that there is a subtle problem in the theoretical description of such processes. Fisher, Nieto, and Sandberg [1] argued that it is not possible to define states by applying the unitary time-evolution operator on the vacuum field state. This argument was partially removed by a consideration using Padé approximants [2]. Later on, however, it was shown by Elyutin and Klyshko [3] and Hillery [4] that for $k=3$ and 4, respectively, a divergence occurs in the mean photon number for finite interaction times. This divergence property may be interpreted as an unphysical artifact coming from the improper treatment of the $k$-photon process. In fact, it has been shown that the usual parametric approximation is incorrect in that it neglects the energy transfer and entanglement between the pump and signal mode of the electromagnetic field, which emerges when the pump mode is quantized [5-8]. We note that the possibility of observing $k=3$ nonlinear quantum optical conversion processes in a damped cavity has recently been discussed [9].

While these phenomena are well known and elaborated upon in the context of nonlinear optics, due to recent advances in laser cooling $[10,11]$, state preparation $[12,13]$, and detection [14] of the motional quantum state of single trapped ions, a type of realization of such nonlinear mode couplings became possible. Here the modes are represented by the three-dimensional harmonic center-of-mass oscillations of a single ion in the trap. The nonlinear mode coupling may be realized by appropriate laser irradiation which induces vibrational Raman transitions [15-19]. This opens possibilities to study such mode couplings with an almost perfect system (i.e., the motion of the trapped ion) where the damping of the motion is negligibly small apart from a small heating rate due to technical imperfections [20]. In the Lamb-Dicke regime, where the atomic center-of-mass position is well localized with respect to the wavelengths of the applied laser fields, mode couplings result which are analogous to the optical mode couplings in the parametric approximation. That is, a treatment of the dynamics based on the Lamb-Dicke approximation would reveal an unphysical divergence of the mean number of vibrational quanta for $k$-quantum processes with $k \geqslant 3$.

Whereas for a trapped atom in the Lamb-Dicke regime one obtains a close connection to the parametrically approximated optical couplings, for a trapped atom, which is not well localized with respect to the laser wavelengths, nonlinear modifications of the couplings occur which arise from the overlap of the atomic center-of-mass wave function with the laser waves, describing the momentum transfer onto the atomic center-of-mass during laser-photon absorption and emission $[15,19]$. These recoil effects may strongly influence the coupling strengths which depend on the number of excited quanta in the vibrational modes. They have been predicted [21] and observed [12] in the context of a nonlinear Jaynes-Cummings model, describing the dynamics of a laser-driven vibronic transition in the resolved-sideband regime.

In this paper we will show that nonlinear effects caused by the overlap of light and matter waves will remove the unphysical divergence problem which arises in the LambDicke approximation. The paper is structured as follows: In Sec. II we introduce the effective Hamiltonian for the motional dynamics of the trapped atom, and we briefly discuss 
$|2\rangle$

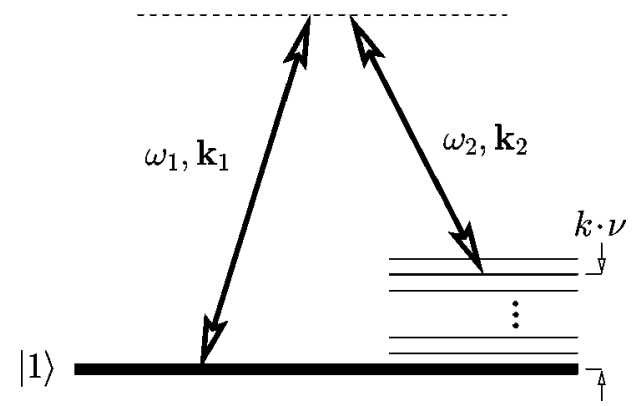

FIG. 1. $k$-quantum motional coupling by application of two offresonant laser fields with laser difference frequency $\omega_{1}-\omega_{2}=k \nu$, where $\nu$ is the frequency of the vibrational mode which is specified by the beam directions $\mathbf{k}_{1}-\mathbf{k}_{2}$ of the beat node of the two lasers.

the justification and the validity of the parametric approximation for optical couplings and the Lamb-Dicke approximation for the motional couplings. The laser-driven motional dynamics is then considered in Sec. III, and the divergence problem in the Lamb-Dicke approximation is studied in Sec. IV. In Sec. V the more realistic treatment of an unspecified degree of localization of the trapped atom is shown to remove the divergence, and some examples for the time evolution are given. A summary and some conclusions are found in Sec. VI.

\section{MOTIONAL COUNTERPART OF MULTIPHOTON DOWN-CONVERSION}

For the $k$-quantum vibrational-mode coupling, we consider here a two-photon vibrational Raman transition which has been experimentally realized $[12,13]$ and theoretically studied in the context of nonlinear couplings of vibrational modes [15-18]. By application of two laser beams which are off resonant with respect to a strong electronic dipole transition, and which are detuned relative to each other by multiples of the vibrational frequencies in the trap, vibrational Raman transitions can be driven which may be used to realize a quantum-mechanical counterpart of nonlinear optics; see Fig. 1. For an appropriate laser-beam propagation geometry which affects only the dynamics in one vibrational mode of frequency $\nu$, in the rotating-wave approximation the Hamiltonian describing the effect of the Raman laser drive on the dynamics of the vibrational mode is given by [15]

$$
\hat{H}_{k}=\hbar \kappa \hat{f}_{k}\left(\hat{a}^{\dagger} \hat{a} ; \eta\right)(i \eta \hat{a})^{k}+\text { H.c. }
$$

where $\hat{a}$ and $\hat{a}^{\dagger}$ are the annihilation and creation operators of vibrational quanta, respectively. Here the laser differencefrequency has been chosen to be $k \nu$, i.e., $k$ times the vibrational frequency of the mode with $k \geqslant 1$ [22]. The effective two-photon coupling strength is given by $\kappa$, and

$$
\eta=2 \pi \frac{\sqrt{\left\langle 0\left|\Delta \hat{x}^{2}\right| 0\right\rangle}}{\lambda}
$$

is the so-called Lamb-Dicke parameter describing the localization of the spatial extension of the center-of-mass wave function in the ground state relative to the wavelength $\lambda$ of the beat node of the two laser beams. The Hermitian operator functions $\hat{f}_{k}\left(\hat{a}^{\dagger} \hat{a} ; \eta\right)$ strongly depend on $\eta$ and are defined by the normally ordered expressions

$$
\begin{aligned}
& \hat{f}_{k}\left(\hat{a}^{\dagger} \hat{a} ; \eta\right)=e^{-\eta^{2} / 2} \sum_{l=0}^{\infty}(-1)^{l} \frac{\eta^{2 l}}{l !(l+k) !} \hat{a}^{\dagger l} \hat{a}^{l} \\
& =:\left(2 \eta \sqrt{\hat{a}^{\dagger} \hat{a}}\right)^{-k} J_{k}\left(\eta \sqrt{\hat{a}^{\dagger} \hat{a}}\right) e^{-\eta^{2} / 2}: .
\end{aligned}
$$

These nonlinear operator functions correspond to overlap integrals of the motional states $|n\rangle$ and $|n+k\rangle$ and the beat node of the laser fields. They account for the recoil effects during the process of absorption and emission of laser photons of the trapped atom. Since they depend only on the quantum number $\hat{n}=\hat{a}^{\dagger} \hat{a}$, in the basis of its eigenstates, $\hat{n}|n\rangle=n|n\rangle \quad(n=0,1,2, \ldots)$, these operators are diagonal, with their diagonal elements $f_{k}(n ; \eta)=\left\langle n\left|\hat{f}_{k}\left(\hat{a}^{\dagger} \hat{a} ; \eta\right)\right| n\right\rangle$ being given by

$$
f_{k}(n ; \eta)=\frac{n !}{(n+k) !} L_{n}^{(k)}\left(\eta^{2}\right) e^{-\eta^{2} / 2},
$$

where $L_{n}^{(k)}(x)$ are the associated Laguerre polynomials. For a well-localized atom, that is, for very small Lamb-Dicke parameters $\eta \ll 1$, or more precisely for a small spatial extension of the atomic wave function $\eta \sqrt{n+1} \ll 1$, one reaches the so-called Lamb-Dicke limit. Here usually the LambDicke approximation is made, which takes into account only the lowest-order terms in $\eta$. In our description of the $k$ quantum coupling [Eq. (1)] the Lamb-Dicke approximation is performed by replacing the operator-valued function $\hat{f}_{k}\left(\hat{a}^{\dagger} \hat{a} ; \eta\right)$ by its limiting value for a small Lamb-Dicke parameter,

$$
\lim _{\eta \rightarrow 0} \hat{f}_{k}(\hat{n} ; \eta)=\frac{1}{k !} .
$$

By replacing the operator function $\hat{f}_{k}(\hat{n} ; \eta)$ in Hamiltonian (1) by the $c$ number given in Eq. (4), one obtains the interaction Hamiltonian $\hat{H}_{k}^{(\mathrm{LD})}$ of the $k$-quantum process in the Lamb-Dicke approximation, that is, in lowest order of the Lamb-Dicke parameter,

$$
\hat{H}_{k}^{(\mathrm{LD})}=\hbar \kappa_{k} \hat{a}^{k}+\hbar \kappa_{k}^{*} \hat{a}^{\dagger k},
$$

with

$$
\kappa_{k}=\kappa \frac{(i \eta)^{k}}{k !}
$$

being the $k$-quantum coupling strength in the Lamb-Dicke approximation.

In the context of nonlinear optics, Hamiltonian (5) describes the $k$-photon down-conversion process, where $\hat{a}$ is the signal mode and the pump mode has been parametrically approximated by replacing its operators by amplitudes whose values are included in the coupling strength $\kappa_{k}$. It is well known that for $k>2$ the parametric approximation described 
by Hamiltonian (5) leads to a divergent behavior of the mean quantum number in the signal mode for finite interaction times, i.e.,

$$
\lim _{t_{2}-t_{1} \rightarrow \Delta t_{\infty}}\left\langle\hat{n}\left(t_{2}\right)\right\rangle-\left\langle\hat{n}\left(t_{1}\right)\right\rangle=\infty,
$$

for a defined interaction time $0<\Delta t_{\infty}<\infty$. In nonlinear optics the parametric approximation of the pump mode fails due to pump depletion and the entanglement of signal and pump modes which is essential in this type of interaction, leading to a energy conservation of the total number of photons in the pump and signal mode [1]. The parametric approximation essentially neglects the energy transfer from the signal to the pump mode, leading to an unbounded increase of the energy in the signal mode. A quantum description of the pump mode is therefore required, regardless of how strong the pump field actually is compared with the signal mode [5-8].

For the case of a single trapped atom, the coupling strength $\kappa_{k}$ contains the classically approximated field amplitudes of the two Raman lasers. While in nonlinear optics the parametric approximation for the pump mode fails, it is expected that for a Raman-driven trapped atom it is the Lamb-Dicke approximation, rather than the replacement of the laser-field operators by their classical amplitudes, which leads to a divergent dynamics. Note that the validity of the Lamb-Dicke approximation is, in principle, in contradiction with a divergent motional excitation in the trap. Eigenstates of the trap potential should only be populated for $\eta \sqrt{n+1}$ $\ll 1$, that is, for higher excitations the approximation is no longer valid, and a diverging mean excitation violates this requirement.

Therefore in all cases, even for a trapped atom with small Lamb-Dicke parameter ( $\eta \ll 1$ ), we have to consider the full problem including the nonlinear operator functions $\hat{f}_{k}(\hat{n} ; \eta)$ in Hamiltonian (1). As already noted, these operator functions describe the effects of momentum transfer onto the atomic center-of-mass motion during the laser-atom interaction $[15,19,21]$. They are of particular importance for higher vibrational excitations $\eta \sqrt{n+1}>1$, and they are discarded in the Lamb-Dicke approximation.

\section{EQUATIONS OF MOTION}

To study the time evolution of the mean quantum number $\langle\hat{n}(t)\rangle$, we will start by deriving from Hamiltonian (1) the equations of motion for the populations of the vibrational levels. The Schrödinger equation is given by

$$
i \hbar \frac{\partial}{\partial t}|\psi(t)\rangle=\hat{H}_{k}|\psi(t)\rangle
$$

where $|\psi(t)\rangle$ is the state vector in the interaction picture. Using as basis the number states $|n\rangle$, we obtain the following equation of motion for the coefficients $\psi_{n}(t)$ $=\langle n \mid \psi(t)\rangle$ :

$$
\frac{\partial \psi_{n}}{\partial t}=-i\left[g_{k}(n ; \eta) \psi_{n+k}+g_{k}^{*}(n-k ; \eta) \psi_{n-k}\right]
$$

where $g_{k}(n ; \eta)$ is given by

$$
g_{k}(n ; \eta)=\kappa(i \eta)^{k} \sqrt{\frac{(n+k) !}{n !}} f_{k}(n ; \eta),
$$

and $g_{k}(n ; \eta)=0$ for $n<0$. For notational simplicity we will omit here and in the following the time argument of $\psi_{n}(t)$ and will only write $\psi_{n}$.

The time evolution of the populations of the number states, $P_{n}=\psi_{n}^{*} \psi_{n}$, is obtained from Eq. (8) and its complex conjugate,

$$
\frac{\partial P_{n}}{\partial t}=2 \operatorname{Im}\left[g_{k}(n ; \eta) \psi_{n}^{*} \psi_{n+k}-g_{k}(n-k ; \eta) \psi_{n-k}^{*} \psi_{n}\right]
$$

To calculate the second time derivative of Eq. (10), one requires the time derivatives of combinations of the type $\psi_{n}^{*} \psi_{n+k}$ which are given by

$$
\begin{aligned}
\frac{\partial}{\partial t} \psi_{n}^{*} \psi_{n+k}= & i g_{k}^{*}(n ; \eta)\left(P_{n+k}-P_{n}\right) \\
& +i\left[g_{k}(n-k ; \eta) \psi_{n-k}^{*} \psi_{n+k}\right. \\
& \left.-g_{k}(n+k ; \eta) \psi_{n}^{*} \psi_{n+2 k}\right] .
\end{aligned}
$$

Inserting Eq. (11) into the time-derivated Eq. (10) the second time derivative of the number statistics results to be

$$
\begin{aligned}
\frac{\partial^{2} P_{n}}{\partial t^{2}}= & 2\left|g_{k}(n ; \eta)\right|^{2}\left(P_{n+k}-P_{n}\right)-2\left|g_{k}(n-k ; \eta)\right|^{2} \\
& \times\left(P_{n}-P_{n-k}\right)-2 \operatorname{Re}\left[g_{k}(n ; \eta) g_{k}(n+k ; \eta) \psi_{n+2 k}^{*} \psi_{n}\right. \\
& +g_{k}(n-k ; \eta) g_{k}(n-2 k ; \eta) \psi_{n}^{*} \psi_{n-2 k} \\
& \left.-2 g_{k}(n ; \eta) g_{k}(n-k ; \eta) \psi_{n+k}^{*} \psi_{n-k}\right] .
\end{aligned}
$$

We are interested here in the temporal evolution of the mean quantum number

$$
\frac{d^{2}\langle\hat{n}(t)\rangle}{d t^{2}}=\sum_{n=0}^{\infty} n \frac{\partial^{2} P_{n}(t)}{\partial t^{2}}
$$

which can be calculated with the help of Eq. (12). Here only the first two terms of Eq. (12) contribute to the sum in Eq. (13), whereas the real part given in Eq. (12) cancels. The resulting equation of motion for the number statistics reads as

$$
\frac{d^{2}\langle\hat{n}(t)\rangle}{d t^{2}}=2 k \sum_{n=0}^{\infty}\left[\left|g_{k}(n ; \eta)\right|^{2}-\left|g_{k}(n-k ; \eta)\right|^{2}\right] P_{n}(t) \text {. }
$$

Defining the coefficients $F_{k}(n ; \eta)$ by the relation

$$
\eta^{2 k}|\kappa|^{2} F_{k}(n ; \eta)=k\left[\left|g_{k}(n ; \eta)\right|^{2}-\left|g_{k}(n-k ; \eta)\right|^{2}\right] \text {, }
$$

one obtains, for Eq. (14), 


$$
\frac{d^{2}\langle\hat{n}(\tau)\rangle}{d \tau^{2}}=\sum_{n=0}^{\infty} F_{k}(n ; \eta) P_{n}(\tau)
$$

with the (dimensionless) scaled time $\tau$ given by

$$
\tau=\sqrt{2} \eta^{k}|\kappa| t
$$

The coefficients $F_{k}(n ; \eta)$ determine the motional dynamics and especially the existence of a divergence in finite interaction times, as depicted in Eq. (6). From Eqs. (9) and (15) the coefficients follow as

$$
F_{k}(n ; \eta)=k\left[\frac{(n+k) !}{n !} f_{k}^{2}(n ; \eta)-\frac{n !}{(n-k) !} f_{k}^{2}(n-k ; \eta)\right]
$$

with $k \geqslant 1$ and the functions $f_{k}(n ; \eta)$ given by Eq. (3), with $f_{k}(n ; \eta)=0$ for $n<0$.

\section{LAMB-DICKE APPROXIMATION: EXPLODING SOLUTIONS}

In the Lamb-Dicke approximation $(\eta \rightarrow 0)$, the coefficients $F_{k}(n ; \eta)$ read as

$$
F_{k}(n ; 0)=\frac{1}{(k-1) !}\left[\left(\begin{array}{c}
n+k \\
k
\end{array}\right)-\left(\begin{array}{l}
n \\
k
\end{array}\right)\right] .
$$

From Eq. (19) it can be seen that, in general, the functions $F_{k}(n ; 0)$ are polynomials in $n$ of the order $k-1$, that is,

$$
F_{k}(n ; 0)=\sum_{l=0}^{k-1} a_{k l} n^{l}
$$

with a nonvanishing highest-order coefficient $a_{k, k-1} \neq 0$. From Eq. (19) it can be seen that the expansion coefficients are always positive $a_{k l} \geqslant 0$. Moreover, the lowest-order coefficient $a_{k 0}$ is nonvanishing, since

$$
a_{k 0}=F_{k}(0 ; 0)=\frac{1}{(k-1) !}>0
$$

The second-order differential equations for the mean excitation number in the Lamb-Dicke approximation [Eq. (16)], together with Eq. (20), read as

$$
\frac{d^{2}\langle\hat{n}(\tau)\rangle}{d \tau^{2}}=\sum_{l=0}^{k-1} a_{k l}\left\langle\hat{n}^{l}(\tau)\right\rangle .
$$

Note, that the second derivative [Eq. (22)] is always positive and nonzero due to the nonvanishing lowest-order term $a_{k 0}$, cf. Eq. (21). For obtaining a lower bound for the second derivative we use the relations following from the Schwarz inequality

$$
\left\langle\hat{n}^{l}(\tau)\right\rangle \geqslant\langle\hat{n}(\tau)\rangle^{l}, \quad l=0,1,2, \ldots
$$

Due to the positiveness of the coefficients $a_{k l}$, a lower bound of the right-hand side of Eq. (22) follows by using Eq. (23),

$$
\frac{d^{2}\langle\hat{n}(\tau)\rangle}{d \tau^{2}}=\sum_{l=0}^{k-1} a_{k l}\left\langle\hat{n}^{l}(\tau)\right\rangle \geqslant \sum_{l=0}^{k-1} a_{k l}\langle\hat{n}(\tau)\rangle^{l}>0
$$

From the formal solution of Eq. (22) and the application of Eq. (24), one obtains the inequality

$$
\langle\hat{n}(\tau)\rangle=\bar{n}_{0}+\bar{n}_{0}^{\prime} \tau+\int_{0}^{\tau} d \tau^{\prime} \int_{0}^{\tau^{\prime}} d \tau^{\prime \prime} \frac{d^{2}\left\langle\hat{n}\left(\tau^{\prime \prime}\right)\right\rangle}{d \tau^{2}} \geqslant \bar{n}_{0}+\bar{n}_{0}^{\prime} \tau+\int_{0}^{\tau} d \tau^{\prime} \int_{0}^{\tau^{\prime}} d \tau^{\prime \prime} \sum_{l=0}^{k-1} a_{k l}\left\langle\hat{n}\left(\tau^{\prime \prime}\right)\right\rangle^{l}
$$

with the initial conditions

$$
\bar{n}_{0}=\left.\langle\hat{n}(\tau)\rangle\right|_{\tau=0}, \quad \bar{n}_{0}^{\prime}=\left.\frac{d\langle\hat{n}(\tau)\rangle}{d \tau}\right|_{\tau=0},
$$

where we have chosen, without loss of generality, the initial time to be $\tau=0$. From Eq. (25), it follows that the solution $N_{\mathrm{lb}}(\tau)$ of the second-order differential equation

$$
\frac{d^{2} N_{\mathrm{lb}}(\tau)}{d \tau^{2}}=\sum_{l=0}^{k-1} a_{k l} N_{\mathrm{lb}}^{l}(\tau)
$$

obeys the relation

$$
N_{\mathrm{lb}}(\tau) \leqslant\langle\hat{n}(\tau)\rangle
$$

for $\tau \geqslant 0$ and identical initial conditions $N_{\mathrm{lb}}(0)=\bar{n}_{0}$ and $N_{\mathrm{lb}}^{\prime}(0)=\bar{n}_{0}^{\prime}$. That is, $N_{\mathrm{lb}}(\tau)$ represents a lower-bound (lb) for the solution $\langle\hat{n}(\tau)\rangle$. We will show in the following that, for $k \geqslant 3$, the lower-bound solution $N_{\mathrm{lb}}(\tau)$ may diverge for finite interaction times, so that it is proved that the correct solution $\langle\hat{n}(\tau)\rangle$ also diverges.

We obtain a first-order differential equation by considering the first derivative $N_{\mathrm{lb}}^{\prime}(\tau)=d N_{\mathrm{lb}}(\tau) / d \tau$,

$$
\frac{d N_{\mathrm{lb}}^{\prime}}{d \tau}=\frac{d N_{\mathrm{lb}}^{\prime}}{d N_{\mathrm{lb}}} \frac{d N_{\mathrm{lb}}}{d \tau}=\frac{d N_{\mathrm{lb}}^{\prime}}{d N_{\mathrm{lb}}} N_{\mathrm{lb}}^{\prime}=\sum_{l=0}^{k-1} a_{k l} N_{\mathrm{lb}}^{l} .
$$

The last equality in Eq. (29) can then be easily solved by integration of

$$
N_{\mathrm{lb}}^{\prime} d N_{\mathrm{lb}}^{\prime}=\sum_{l=0}^{k-1} a_{k l} N_{\mathrm{lb}}^{l} d N_{\mathrm{lb}}
$$

and one obtains 


$$
\left[\frac{d N_{\mathrm{lb}}(\tau)}{d \tau}\right]^{2}=\bar{n}_{0}^{\prime 2}+\sum_{l=1}^{k} b_{k l}\left[N_{\mathrm{lb}}^{l}(\tau)-\bar{n}_{0}^{l}\right]
$$

with the coefficients $b_{k l}=2 a_{k, l-1} / l \geqslant 0$ and $b_{k 1} \neq 0, b_{k k} \neq 0$.

To demonstrate the unphysical properties of the Hamiltonian $\hat{H}_{k}^{(\mathrm{LD})}$ for $k \geqslant 3$ in the Lamb-Dicke approximation (5), it is sufficient to prove the unphysical behavior for one physically reasonable initial condition. For the special cases $k=3$ and 4 , this has already been explicitly shown in Refs. $[3,4]$; here we want to show the unphysical behavior, in a general way, for all $k \geqslant 3$. For the atom initially (at $\tau=0$ ) in its vibrational ground state, $|\psi(0)\rangle=|0\rangle$, the initial conditions are $\bar{n}_{0}=\bar{n}_{0}^{\prime}=0$ [the latter can be seen from Eq. (10) with $\left.\psi_{n}(0)=\delta_{n, 0}\right]$ and the differential equation (31) reduces to

$$
\frac{d N_{\mathrm{lb}}(\tau)}{d \tau}=\left[\sum_{l=1}^{k} b_{k l} N_{\mathrm{lb}}^{l}(\tau)\right]^{1 / 2} .
$$

Here we have chosen the positive square root, since for vanishing initial velocity $\bar{n}_{0}^{\prime}=0$ and always positive acceleration $d^{2} N_{\mathrm{lb}}(\tau) / d \tau^{2}>0$ [cf. Eqs. (24) and (27)], the velocity at time $\tau>0$ has to be positive: $d N_{\mathrm{lb}}(\tau) / d \tau>0$. Equation (32) can then be integrated from the finite time $\tau_{1}>0$ to $\tau_{2} \geqslant \tau_{1}$ which gives the relation

$$
\tau_{2}-\tau_{1}=\int_{N_{\mathrm{lb}}\left(\tau_{1}\right)}^{N_{\mathrm{lb}}\left(\tau_{2}\right)} \frac{d n}{\sqrt{b_{k k} n^{k}+\cdots+b_{k 2} n^{2}+b_{k 1} n^{1}}} .
$$

Since the velocity $d N_{\mathrm{lb}}(\tau) / d \tau$ is always positive and nonvanishing for $\tau>0$, it is clear that $N_{\mathrm{lb}}\left(\tau_{1}\right)>\bar{n}_{0}=0$. Therefore the integration starts with a positive and nonvanishing value of the excitation, $N_{\mathrm{lb}}\left(\tau_{1}\right)>0$, that has been attained after the interaction time $\tau_{1}$.

Now we are interested in the further evolution in the time interval $\tau_{2}-\tau_{1}$. In particular, we are looking for that time interval $\Delta \tau_{\infty}=\tau_{2}-\tau_{1}$ for which the excitation number $N_{\mathrm{lb}}\left(\tau_{2}\right)$ attains an infinite value $N_{\mathrm{lb}}\left(\tau_{2}\right) \rightarrow \infty$. By taking only the highest-order term in the square-root of Eq. (33) we obtain an upper bound for $\Delta \tau_{\infty}$ :

$$
\Delta \tau_{\infty} \leqslant \int_{N_{\mathrm{lb}}\left(\tau_{1}\right)}^{\infty} \frac{d n}{\sqrt{b_{k k} n^{k}}}=\left\{\begin{array}{cc}
\infty & (k=1,2) \\
\frac{2}{k-2} \frac{1}{\sqrt{b_{k k} N_{\mathrm{lb}}^{k-2}\left(\tau_{1}\right)}} & (k \geqslant 3) .
\end{array}\right.
$$

This result reveals that we obtain a finite value of $\Delta \tau_{\infty}$ for $k \geqslant 3$. That is, after attaining the finite (nonvanishing) excitation $N_{\mathrm{lb}}\left(\tau_{1}\right)$ after the interaction time $\tau_{1}$, the solution $N_{\mathrm{lb}}(\tau)$ of Eq. (27) already diverges after the finite time interval $\Delta \tau_{\infty}$, according to Eq. (34). Concluding, for $k \geqslant 3$ the solution of Eq. (22) $\left[\left\langle\hat{n}\left(\tau_{2}\right)\right\rangle \geqslant N_{\mathrm{lb}}\left(\tau_{2}\right)\right]$ will also diverge at a certain finite interaction time $\tau_{2} \leqslant \tau_{1}+\Delta \tau_{\infty}$. For $k=1$ and 2 , no upper bound for the interaction time can be given, and it can be seen by direct integration of Eq. (22) that the mean excitation does not diverge in a finite interaction time.

\section{OVERLAP OF MATTER AND LIGHT WAVES: REGULAR BEHAVIOR FOR LARGE EXCITATIONS}

In Sec. IV it has been shown that in the Lamb-Dicke approximation the mean motional excitation number diverges in a finite interaction time for the cases $k \geqslant 3$. In this section we will prove that the exact Hamiltonian (1), e.g., without the Lamb-Dicke approximation, does not exhibit such a divergence problem. This is due to the overlap of matter and light waves described by the nonlinear operator functions (2). They lead to an excitation-dependent coupling strength which suppresses the unbounded increase of the mean excitation.

\section{A. Proof of the regular behavior}

To prove the regular behavior of the dynamics of the system described by Eqs. (16) and (18) we may consider the following situations. (a) If the mean quantum number diverges, we would be operating in a regime of very large quantum numbers $n$. Therefore we are allowed to use an asymptotic expansion of the coefficients $F_{k}(n ; \eta)$ for large $n$.

(b) Since for $k=1$ and 2 we know that in the Lamb-Dicke approximation [described by $F_{1,2}(n ; 0)$ ] the dynamics does not exhibit a divergence in finite interaction times, it is sufficient to show that the asymptotic expansion of $F_{k}(n ; \eta)$ has an upper bound leading to a dynamics which is at least as convergent as for $F_{1,2}(n ; 0)$,

$$
F_{k}(n ; \eta) \leqslant F_{1,2}(n ; 0) \quad(n \gg 1) .
$$

Then the acceleration $d^{2}\langle\hat{n}(\tau)\rangle / d \tau^{2}$ is always smaller than those for the well-behaved cases, and a divergence in finite times cannot exist, regardless of the initial motional quantum state chosen.

We start by expressing the function $F_{k}(n ; \eta)$ given in Eq. (18) in terms of Laguerre polynomials by using Eq. (3),

$$
\begin{aligned}
F_{k}(n ; \eta)= & \left\{\frac{n !}{(n+k) !}\left[L_{n}^{(k)}\left(\eta^{2}\right)\right]^{2}\right. \\
& \left.-\frac{(n-k) !}{n !}\left[L_{n-k}^{(k)}\left(\eta^{2}\right)\right]^{2}\right\} e^{-\eta^{2} / 2} .
\end{aligned}
$$

While the first (positive) term in Eq. (36) describes the transition to higher-lying states $|n\rangle \rightarrow|n+k\rangle$, the second (nega- 
tive) term describes transitions to lower-lying states $|n\rangle$ $\rightarrow|n-k\rangle$, leading to a decrease of the acceleration. An upper bound for $F_{k}(n ; \eta)$, which determines the maximum $a c$ celeration, is therefore given by neglecting the transitions to lower-lying states (which do not cause a divergent behavior),

$$
F_{k}(n ; \eta) \leqslant \frac{n !}{(n+k) !}\left[L_{n}^{(k)}\left(\eta^{2}\right)\right]^{2} e^{-\eta^{2} / 2}
$$

Using the relation between the Laguerre polynomials and the confluent hypergeometric (Kummer's) function $M(a, b ; x)$ [23],

$$
L_{n}^{(k)}(x)=\left(\begin{array}{c}
n+k \\
n
\end{array}\right) M(-n, k+1 ; x),
$$

one arrives at the inequality for $F_{k}(n ; \eta)$

$$
F_{k}(n ; \eta) \leqslant \frac{1}{k !}\left(\begin{array}{c}
n+k \\
k
\end{array}\right) M^{2}\left(-n, k+1 ; \eta^{2}\right) e^{-\eta^{2} / 2}
$$

An asymptotic expansion of the confluent hypergeometric function $M(a, b ; x)$ for $a \rightarrow-\infty$, bounded $b$, and real-valued $x$ is given by [23]

$$
\begin{aligned}
M(a, b ; x) \sim & \frac{\Gamma(b)}{\sqrt{\pi}} e^{(1 / 2) x\left[\left(\frac{b}{2}-a\right) x\right]^{(1 / 4)-(1 / 2) b}} \\
& \times \cos \left[\sqrt{(2 b-4 a) x}-\frac{1}{2} b \pi+\frac{1}{4} \pi\right] .
\end{aligned}
$$

Thus, for large numbers $n$, the inequality in its asymptotic form reads

$$
\begin{aligned}
F_{k}(n ; \eta) \leqslant & \frac{1}{\pi} \frac{(n+k) !}{n !}\left[\eta^{2}\left(n+\frac{1+k}{2}\right)\right]^{-k-(1 / 2)} \\
& \times \cos ^{2}\left[2 \eta \sqrt{n+\frac{1+k}{2}}-\frac{1}{2} k \pi-\frac{1}{4} \pi\right] e^{\eta^{2} / 2}
\end{aligned}
$$

Therefore, the maximum value of the right-hand side of Eq. (41) can be estimated by taking the squared cosines to be unity,

$$
F_{k}(n ; \eta) \leqslant \frac{(n+k) !}{\pi n !}\left[\eta^{2}\left(n+\frac{1+k}{2}\right)\right]^{-k-(1 / 2)} e^{\eta^{2} / 2}
$$

Moreover, expression (42) can be further estimated by the relation

$$
\frac{(n+k) !}{n !}=(n+k)(n+k-1) \cdots(n+1) \leqslant(n+k)^{k} \text {, }
$$

which gives one a further simplification:

$$
F_{k}(n ; \eta) \leqslant \frac{1}{\pi} e^{\eta^{2} / 2} \frac{1}{\sqrt{\eta^{2}\left(n+\frac{1+k}{2}\right)}}\left[\frac{(n+k)}{\eta^{2}\left(n+\frac{1+k}{2}\right)}\right]^{k}
$$

For the range of large numbers $n$, we are considering here, the function therefore has the upper bound

$$
F_{k}(n ; \eta) \leqslant \frac{1}{\pi} \frac{e^{\eta^{2} / 2}}{\eta^{2 k+1}} \frac{1}{\sqrt{n}}
$$

that is, for large numbers $n$, the upper bound of the function $F_{k}(n ; \eta)$ decays as $1 / \sqrt{n}$. It therefore can be further estimated by a simple constant $C_{k}(\eta)$,

$$
F_{k}(n ; \eta) \leqslant C_{k}(\eta), \quad C(\eta)=\frac{1}{\pi} \frac{e^{\eta^{2} / 2}}{\eta^{2 k+1}} .
$$

The resulting differential equation for the upper bound (ub) $N_{\mathrm{ub}}(\tau)$ of the mean quantum number reduces then for possibly large numbers $n$ to

$$
\frac{d^{2} N_{\mathrm{ub}}(\tau)}{d \tau^{2}}=C_{k}(\eta)
$$

Reconsidering the formal solution [Eq. (25)] and the upperbound acceleration [Eq. (46)], it becomes clear that $N_{\mathrm{ub}}(\tau)$ indeed is an upper bound for the exact mean excitation number

$$
N_{\mathrm{ub}}(\tau) \geqslant\langle\hat{n}(\tau)\rangle
$$

for identically chosen initial conditions $N_{\mathrm{ub}}(0)=\bar{n}_{0}$ and $N_{\mathrm{ub}}^{\prime}(0)=\bar{n}_{0}^{\prime}$ and large excitations $\langle\hat{n}(\tau)\rangle \gg 1$. Equation (47) states that the mean excitation number does not diverge in finite time, since the differential equation for large values of $n$ leads to a behavior which is as convergent as in the case of $k=1$ in the Lamb-Dicke limit where $F_{1}(n ; 0)=1=$ const. That is, the upper-bound solution of Eq. (47), $N_{\mathrm{ub}}(\tau)$, which can be obtained by direct integration,

$$
N_{\mathrm{ub}}(\tau)=\bar{n}_{0}+\bar{n}_{0}^{\prime} \tau+\frac{1}{2} C_{k}(\eta) \tau^{2}
$$

does not diverge for finite interaction times $\tau$. In conclusion, it has been proved that the mean motional excitation number resulting from the full Hamiltonian (1) does not diverge for finite interaction times.

\section{B. Numerical examples}

As an example, in Fig. 2 we show the exact time evolution of the mean motional excitation number $\langle\hat{n}(\tau)\rangle$ for $k$ $=3$ and the Lamb-Dicke parameter $\eta=0.2$. This clearly shows that, instead of diverging in a finite interaction time as would be expected in the Lamb-Dicke approximation, the mean excitation number exhibits an oscillatory behavior. This is due to the destructive overlap of matter and light waves, leading to a decoupling of the atomic motion from the laser fields for certain excitation amplitudes. 


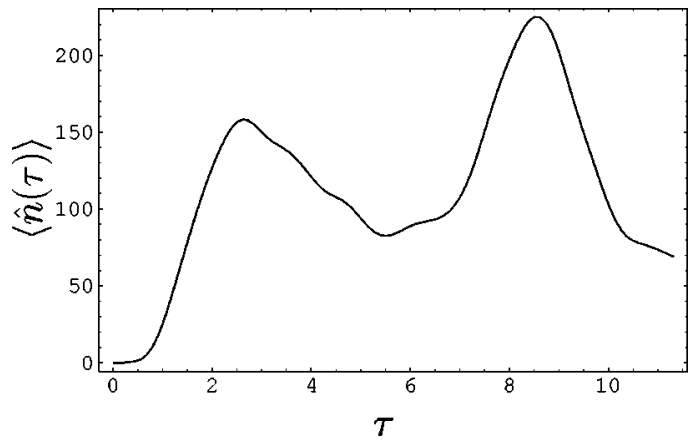

FIG. 2. Exact time evolution of the mean motional excitation number $\langle\hat{n}(\tau)\rangle$ for $k=3$ and Lamb-Dicke parameter $\eta=0.2$, as a function of the scaled time $\tau$ given in Eq. (17).

To gain more insight into the distribution of the quantum state in phase space, in Fig. 3 we show the time evolution of the $Q$ function for the three-quantum coupling $(k=3)$ and for $\eta=0.2$. It can be seen that the dynamics is strongly modified by the occurrence of the circles of vanishing coupling strengths. In contrast to the dynamics in the LambDicke approximation, where the "starlike" structure would be extended to infinitely large phase-space amplitudes, the extension of the star structure is halted at the first circle of vanishing coupling. Parts of the phase-space distribution are smoothed over the circle. For those components of the distribution that accumulate a phase shift of $\pi / 3$ relative to the initial star structure, Hamiltonian (1) effectively exhibits a change of sign accompanied by a reversal of the time evolu- tion. Consequently, those components of the quantum state are moving back toward the origin of phase space [24]. This effect explains the decrease of the mean motional excitation number as seen in Fig. 2. Note that the distribution in Fig. 3(f) for time $\tau=5.74$ corresponds to a local minimum of $\langle\hat{n}(\tau)\rangle$ in Fig. 2. Obviously, there are some components of the phase-space distribution which cross the barrier. However, because of the existence of further barriers at approximately equidistant radii, the explosive dynamics occurring in the Lamb-Dicke approximation and also in the optical parametric approximation does not occur.

\section{SUMMARY AND CONCLUSIONS}

In summary, it has been shown that for a trapped atom which is driven by Raman-laser fields, in the Lamb-Dicke approximation a behavior appears which is analogous to the case of $k$-photon down-conversion in nonlinear optics. A divergent behavior of the mean motional excitation number after finite interaction times occurs for higher-order quantum couplings with $k \geqslant 3$, similar to the situation for the parametric approximation in nonlinear optics. We have discussed these divergences within a single unified framework for all orders $k \geqslant 3$. Moreover, it has been argued that the LambDicke approximation, which is only valid for well-localized atoms, is not consistent with the occurrence of large (or even diverging) mean excitations.

To overcome the divergent behavior, one has to treat the full problem without the Lamb-Dicke approximation. This
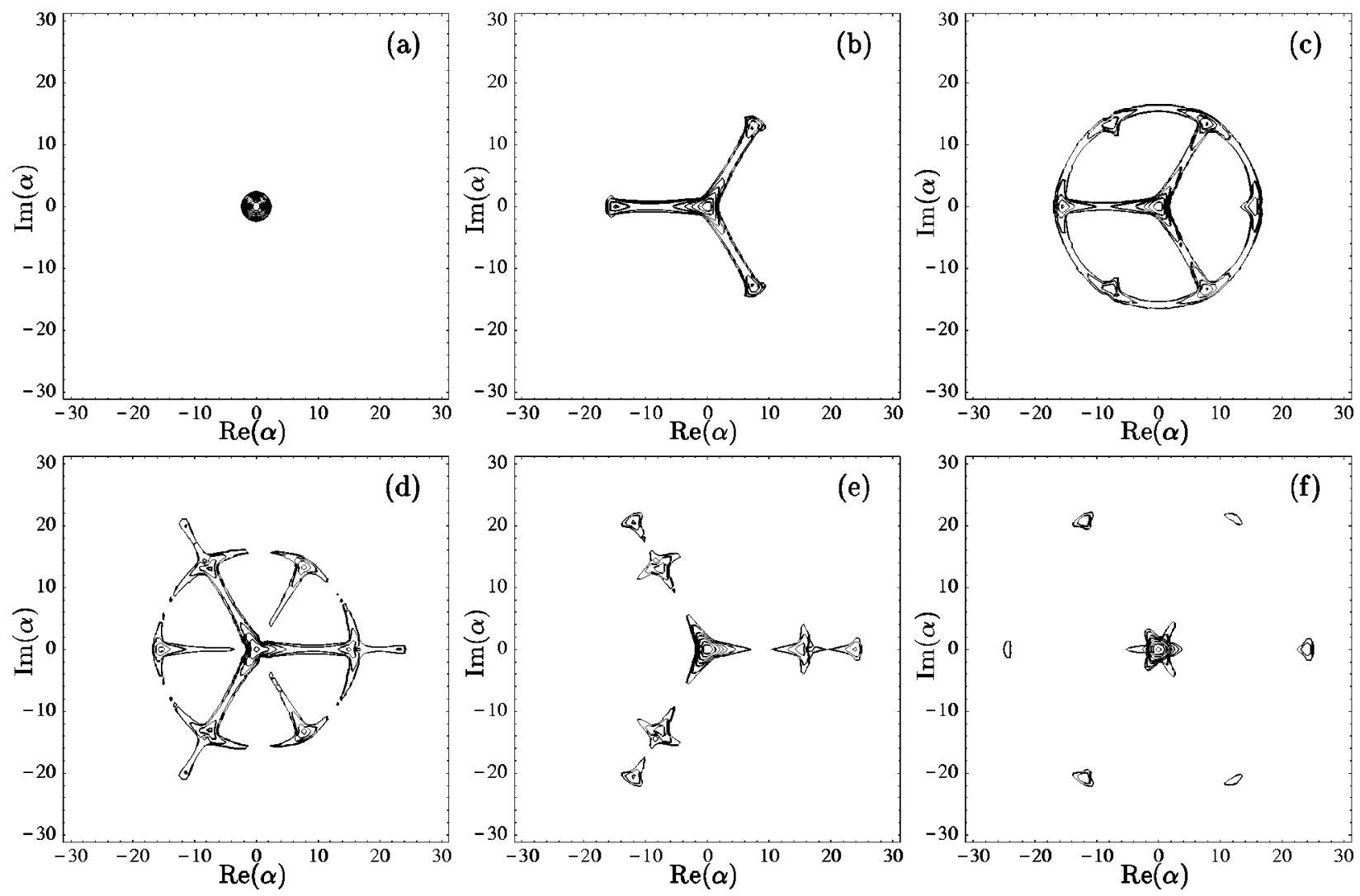

FIG. 3. Time evolution of the Husimi $Q$ function for an initial motional ground state under the influence of the three-quantum coupling $(k=3)$. The Lamb-Dicke parameter has been chosen as $\eta=0.2$, and the scaled times $\tau$ are 0 (a), 1.14 (b), 2.29 (c), 3.44 (d), 4.59 (e), and 5.74 (f). Note the formation of a "star" followed by a ring from which further structure grows. 
includes the correct description of the laser-induced momentum transfer onto the center-of-mass of the trapped atom. These are described by a nonlinear operator function, which plays an essential role for the dynamics of the motional quantum state of the atom. By using an asymptotic expansion, we have proved that the correct description of the recoil effects widely modifies the dynamics for large excitations, and prevents the mean excitation number from exploding for finite interaction times. That is, the full problem leads to a regular dynamics where the energy of the motional degree of freedom does not unphysically diverge. On the other hand, the Lamb-Dicke approximation fails for these types of couplings, as does the parametric approximation in nonlinear optics. Whereas in nonlinear optics the divergence problem arises from the neglection of the pump-mode depletion and entanglement of the involved field modes, in the case of a trapped atom the unappropriate treatment of the recoil effects in the Lamb-Dicke approximation leads to unphysical behavior.

\section{ACKNOWLEDGMENTS}

This work was supported by the Deutsche Forschungsgemeinschaft, by the UK Engineering and Physical Sciences Research Council, and by the European Union.
[1] R. A. Fisher, M. M. Nieto, and V. D. Sandberg, Phys. Rev. D 29, 1107 (1984).

[2] S. L. Braunstein and R. I. McLachlan, Phys. Rev. A 35, 1659 (1987).

[3] P. V. Elyutin and D. N. Klyshko, Phys. Lett. A 149, 241 (1990).

[4] M. Hillery, Phys. Rev. A 42, 498 (1990).

[5] V. Buźek and G. Drobný, Phys. Rev. A 47, 1237 (1993).

[6] G. Drobný, I. Jex, and V. Buzek, Phys. Rev. A 48, 569 (1993).

[7] G. Drobný and V. Buzek, Phys. Rev. A 50, 3492 (1994).

[8] K. Banaszek and P. L. Knight, Phys. Rev. A 55, 2368 (1997).

[9] T. Felbinger, S. Schiller, and J. Mlynek, Phys. Rev. Lett. 80, 492 (1998).

[10] W. Neuhauser, M. Hohenstatt, P. E. Toschek, and H. G. Dehmelt, Phys. Rev. Lett. 41, 233 (1978); D. Wineland, R. Drullinger, and F. Walls, ibid. 40, 1639 (1978).

[11] F. Diedrich, J. C. Bergquist, W. M. Itano, and D. J. Wineland, Phys. Rev. Lett. 62, 403 (1989); C. Monroe, D. M. Meekhof, B. E. King, S. R. Jefferts, W. M. Itano, and D. J. Wineland, ibid. 75, 4011 (1995).

[12] D. M. Meekhof, C. Monroe, B. E. King, W. M. Itano, and D. J. Wineland, Phys. Rev. Lett. 76, 1796 (1996).

[13] C. Monroe, D. M. Meekhof, B. E. King, and D. J. Wineland, Science 272, 1131 (1996).

[14] D. Liebfried, D. M. Meekhof, B. E. King, C. Monroe, W. M. Itano, and D. J. Wineland, Phys. Rev. Lett. 77, 4281 (1996).
[15] S. Wallentowitz and W. Vogel, Phys. Rev. A 55, 4438 (1997).

[16] G. S. Agarwal and J. Banerji, Phys. Rev. A 55, R4007 (1997).

[17] J. Steinbach, J. Twamley, and P. L. Knight, Phys. Rev. A 56, 4815 (1997).

[18] G. Drobný and B. Hladký, Acta Phys. Slov. 47, 277 (1997).

[19] S. Wallentowitz and W. Vogel, Phys. Rev. A 58, 679 (1998).

[20] D. J. Wineland, C. Monroe, W. M. Itano, D. Liebfried, B. E. King, and D. M. Meekhof, NIST J. Res. 103, 259 (1998).

[21] W. Vogel and R. L. de Matos Filho, Phys. Rev. A 52, 4214 (1995).

[22] For $k=0$, one obtains a zero quantum coupling, where no vibrational quanta are created or annihilated in the motion of the trapped atom. For this case only the excitation-dependent operator function $f_{0}(\hat{n} ; \eta)$ acts on the motional degree of freedom and leads to phase-shift and dispersive effects; for more details see Ref. [15]. We exclude this case here, since we are mainly interested in the particular situation for $k \geqslant 3$.

[23] Handbook of Mathematical Functions, edited by M. Abramowitz and I. A. Stegun (Dover, New York, 1965). (a) For the relation between Laguerre polynomials and the confluent hypergeometric function see Chap. 13, Eq. (13.6.9). (b) For the asymptotic expansion of the confluent hypergeometric function, see Chap. 13, Eq. (13.5.14).

[24] For a more detailed discussion of these phase-locking effects in the case of nonlinear squeezing $(k=2)$, cf. Ref. [19]. 